\title{
EVALUATION OF BRAIN ATROPHY MEASURES IN MRI
}

\author{
M.S. Atkins ${ }^{1}$, J. Orchard ${ }^{1}$, M.K. Tory ${ }^{1}$ \\ ${ }^{1}$ School of Computing Science, Simon Fraser University, Burnaby, B.C., Canada
}

\begin{abstract}
Other researchers have proposed that the brain parenchymal fraction (or brain atrophy) may be a good surrogate measure for disease progression in patients with Multiple Sclerosis. This paper considers various factors influencing the measure of the brain parenchymal fraction obtained from head MRI scans. An automatic segmentation method for the brain and for the cerebral spinal fluid is evaluated and the sensitivity of the brain atrophy measure to various parameters is measured. We show that our automatic method can provide robust, reproducible brain atrophy measures.
\end{abstract}

Keywords - Brain atrophy; MRI; CSF segmentation; ventricular CSF; brain parenchymal fraction

\section{INTRODUCTION}

We are interested in measuring brain atrophy for studying the course of diseases like Multiple Sclerosis (MS). Other researchers have proposed the use of the brain parenchymal fraction $(\mathrm{BPF})$ to measure whole brain atrophy in relapsing-

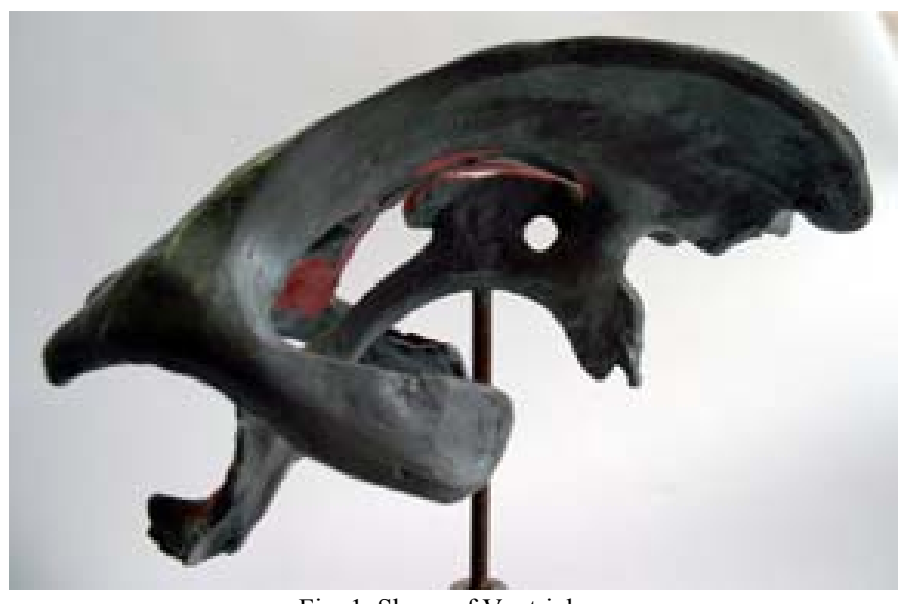

Fig. 1. Shape of Ventricles

remitting MS [1][2], where the BPF is obtained from the intradural volume (IDV) and the cerebral-spinal fluid (CSF) using the simple formula: $\mathrm{BPF}=(\mathrm{IDV}-\mathrm{CSF}) / \mathrm{IDV}$. Follow-up research shows that indeed the BPF, as obtained from the IDV and CSF measured by semi-automatic analysis of PD/T2 dualecho MRI scans, is a reproducible measure [3][4].

We are studying how to obtain a reliable, reproducible measure of brain atrophy automatically from head MRI scans acquired as $5 \mathrm{~mm}$ thick axial slices. We were concerned that the BPF measure could be very sensitive to parameters such as those used in the segmentation of the intra-dural volume (IDV) from the head, and those used in the segmentation of the cerebral spinal fluid (CSF) from the intra-cranial volume.

The measurement of the ventricular cerebral spinal fluid (VCSF) is likely to be sensitive to exact slice location since it is difficult to sample the complex 3D structure of the ventricles accurately using $5 \mathrm{~mm}$ thick slices (see Fig. 1). We hypothesized that despite this issue, it would still be possible to obtain a stable and reliable BPF measure.

This research describes an automatic method for obtaining the BPF from PD/T2 dual-echo MRI scans, and evaluates the sensitivity of the method to various parameters, with the goal of developing a robust, reproducible and versatile automatic method for measuring brain atrophy.

\section{METHODOLOGY}

The data consists of dual spin-echo PD and T2 scans acquired for MS studies with $\mathrm{TR}=3000 \mathrm{~ms}, \mathrm{TE}=30 \mathrm{~ms}$ and $90 \mathrm{~ms}$, slice thickness $=5 \mathrm{~mm}$, and in-plane pixel size of $0.859 \mathrm{~mm}^{2}$. One data set (labeled A0512) consists of a time series of 11 scans of the same MS patient (roughly one scan every month), each with 21 axial slices. The top 3 slices of the brain are missing from this data set, as little MS activity is viewed in these slices. To measure whether the BPF could be calculated from these incomplete brain data sets, we also examined a time series of 8 scans of another patient data set (labeled C1001) with a complete brain in 24 slices, acquired with the same parameters, with a scan every month.

The brain atrophy measures are based on our automatic method for segmenting the brain from the head in MRI scans [5]. The brain mask is obtained by anisotropic diffusion of the T2 image, and thresholding the resulting blurry image. The non-brain areas, such as the eyes, are removed by morphological operations. The intra-dural volume (IDV) is obtained from this mask.

The total cerebral spinal fluid is obtained as described in the first step of our automatic method for isolating MS lesions [6]. As a preprocessing step, both the PD and the T2 images were normalized, using the initial brain mask as a region of interest for the head images. The voxel intensities within 3 standard deviations from the mean intensity under the brain mask were remapped to the range $[0,255]$, as described in [7]. To find the CSF from the normalized brain images, we calculated the ratio image PD/T2 and applied a threshold to extract the CSF. In the ratio image, the CSF appeared dark since the CSF was very bright in T2-weighted MR images.

We tested the sensitivity of the BPF measurements with respect to three major parameters: the threshold on the ratio image in the calculation of the CSF volume, the amount of ventricular CSF (VCSF) measured, and the outline for the intradural brain mask.

The IDV depends on the brain mask, so measures of the IDV alone would require very accurate thresholding of the head image. Our automatic method is $100 \%$ reproducible, and has been assessed to be acceptably accurate [5], but absolute 
measures of the IDV are dependent on partial volume effects, especially for thick slices.

The CSF volume measurements depend on the threshold used on the ratio image $\mathrm{PD} / \mathrm{T} 2$. The total CSF volume was measured when the ratio image $\mathrm{PD} / \mathrm{T} 2$ was thresholded at different values, from 0.6 to 0.9 . These CSF volumes were then used to calculate the BPF, and so the sensitivity of the BPF to the threshold was measured.

The calculation of the sensitivity of the CSF to the amount of ventricular CSF required segmentation of the VCSF from the total CSF. For this study, we employed a (temporary) manual outline on the CSF mask to isolate the ventricular CSF from the total CSF. We then calculated the total VCSF in each scan to determine if the slice positioning could have a deleterious effect on the repeatability of the CSF measure.

The sensitivity of the IDV and CSF volume (and hence the $\mathrm{BPF}$ ) to the outline of the initial brain mask was measured by performing morphological image erosion and dilation on the original brain mask to create new brain masks. These new masks were used for preprocessing and renormalizing the data prior to obtaining the CSF and BPF.

\section{RESULTS}

\section{A. Intra-dural volume}

The time series of intra-dural volumes of two patients, obtained by automatic segmentation from the head, is plotted in Fig. 2. For patient A0512, the mean IDV $=1225.0 \mathrm{~mL}$, standard deviation $\sigma=14.61 \mathrm{~mL}$, and the coefficient of variation $(\mathrm{CV})=$ $1.2 \%$. For patient $\mathrm{C} 1001$, the mean $\mathrm{IDV}=1059.7 \mathrm{~mL}$, standard deviation $\sigma=5.15 \mathrm{~mL}$, and the $\mathrm{CV}=0.49 \%$.

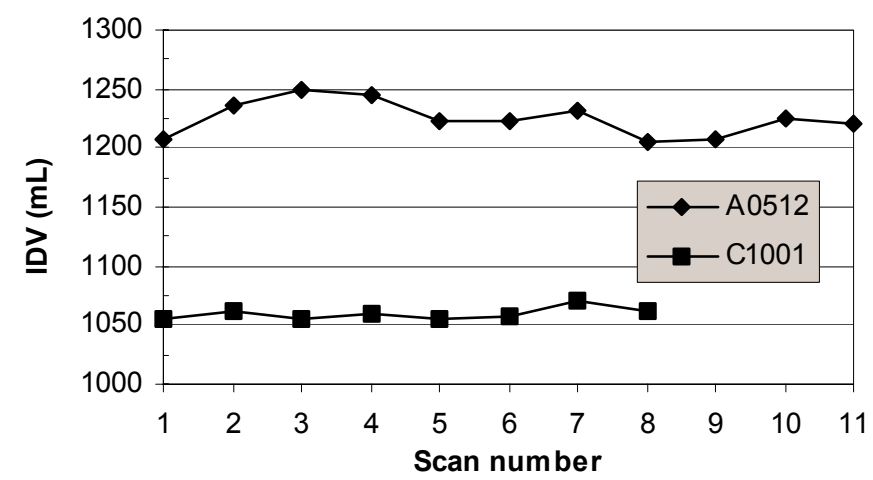

Fig. 2. Intra-Dural Volume versus scan number for patients A0512 and C1001

\section{B. CSF volume}

The volume of CSF (using the ratio image threshold of 0.75 ) for the two patients' time series is plotted in Fig. 3. For patient $\mathrm{A} 0512$, the mean $\mathrm{CSF}=215.8 \mathrm{~mL}$, standard deviation $\sigma=6.04 \mathrm{~mL}$ (assuming no trend) and the $\mathrm{CV}=2.8 \%$. For patient $\mathrm{C} 1001$, the mean $\mathrm{CSF}=167.0 \mathrm{~mL}$, standard deviation $\sigma=3.98 \mathrm{~mL}$, and the $\mathrm{CV}=2.4 \%$.

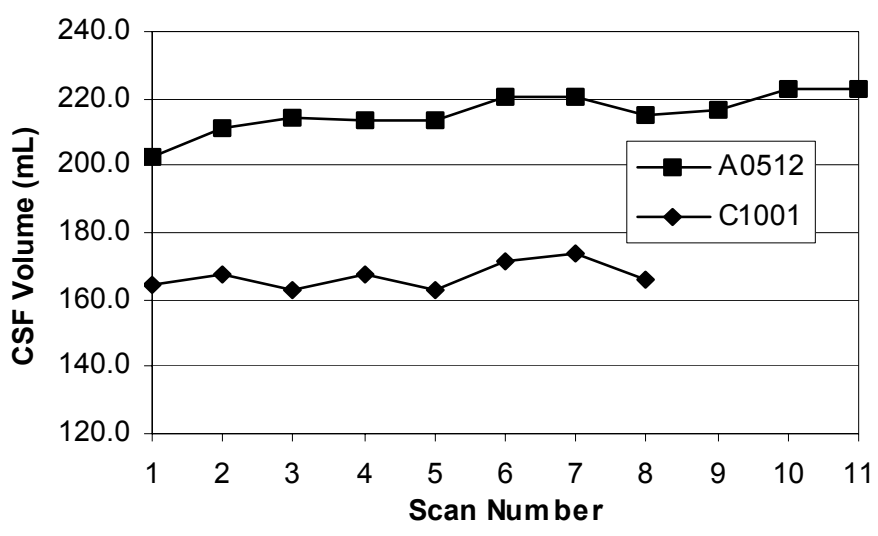

Fig 3. CSF versus scan number for patients A0512 and C1001

\section{Sensitivity of CSF volume to ratio image threshold}

The threshold chosen for the ratio $\mathrm{PD} / \mathrm{T} 2$ affects the CSF volume measured. As the CSF appears light (high intensity) in the T2 image, the CSF appears dark in the ratio image. We found that the CSF occupied the dark pixels in the ratio image slices with intensities below 0.75 . Fig. 4 shows the outline of the CSF mask superimposed on the T2 image for a central brain

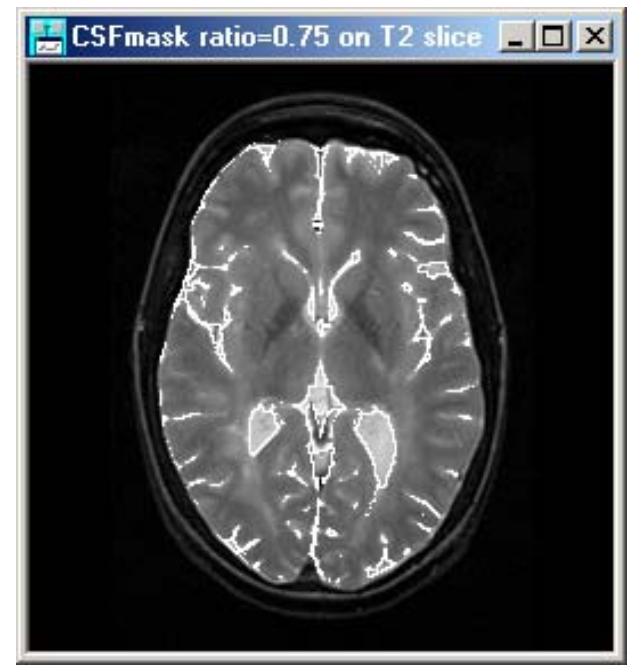

Fig. 4. CSF mask for a central brain slice for a ratio image threshold of 0.75

slice for a threshold of 0.75 .

Raising the threshold increased the number of pixels associated with CSF, and hence raised the apparent volume of CSF, as shown in Fig. 5. Note that the central line in Fig. 5 corresponds to the data in Fig. 3 for C1001.

Correspondingly, increasing the ratio image threshold (which increases the CSF volume) decreases the BPF measure, as seen in Fig. 6. 


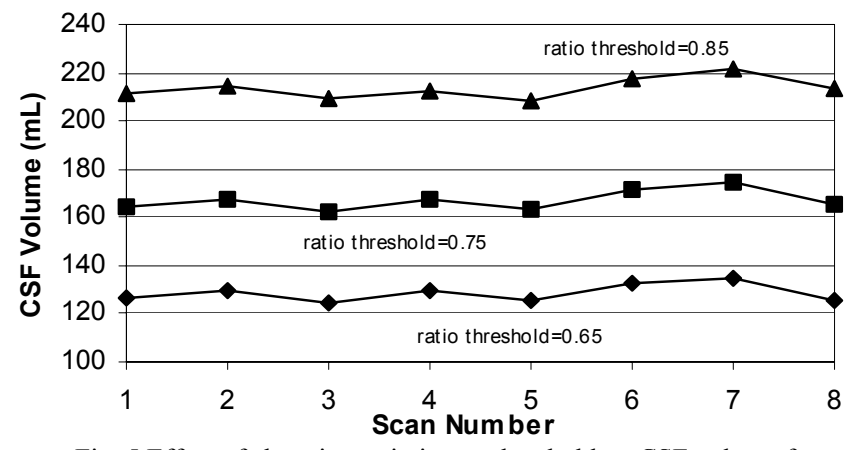

Fig. 5 Effect of changing ratio image threshold on CSF volume for patient $\mathrm{C} 1001$

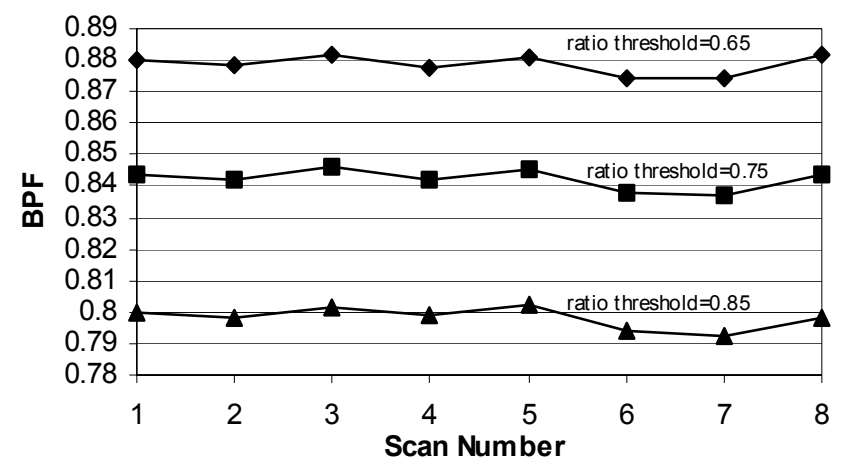

Fig.6. Effect of changing ratio image threshold on BPF for patient C1001

\section{Sensitivity of ventricular CSF to patient position}

The VCSF was measured by drawing a region of interest (ROI) manually around the CSF mask obtained automatically by thresholding the ratio image at 0.75 . The manual ROI corresponds to the lateral and third ventricles in the appropriate slices of each time series scan.

The VCSF volume is plotted in Fig. 7 for the A0512 time series. The mean $\mathrm{VCSF}=53.96 \mathrm{~mL}, \sigma=2.60 \mathrm{~mL}$ and the $\mathrm{CV}=$ $4.8 \%$.

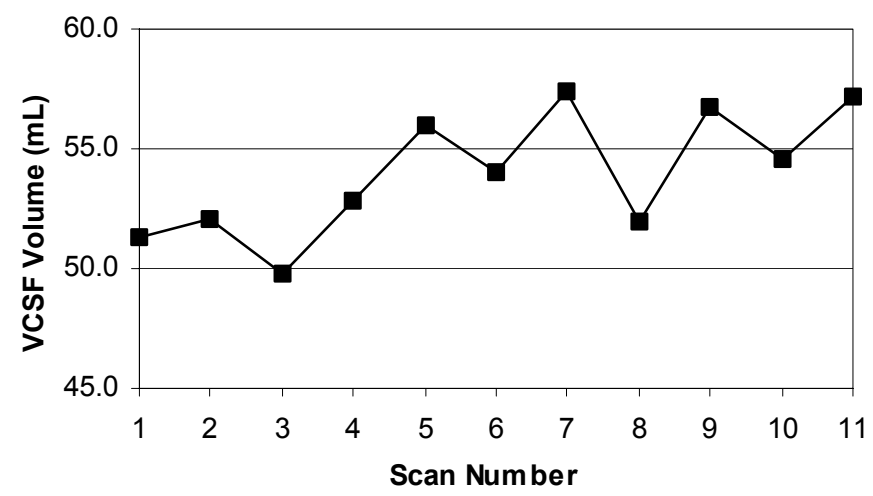

Fig 7. Ventricular CSF volume for patient A0512

\section{E. Brain atrophy measure}

The brain parenchymal fraction for each scan is plotted in Fig. 8 for the two patients. For patient A0215, the mean BPF =
0.824, standard deviation $\sigma=0.0052$ (assuming no trend) and the $\mathrm{CV}=0.63 \%$. Regression analysis shows a significant downward trend of -.00148 per time unit $\left(\mathrm{R}^{2}=90.7 \%\right.$, significance $\mathrm{F}<0.00001$ ). For patient $\mathrm{C} 1001$, the mean $\mathrm{BPF}=$ 0.8424 , standard deviation $\sigma=0.0032$, and the $\mathrm{CV}=0.38 \%$. This patient shows a smaller, insignificant downward trend $\left(\mathrm{R}^{2}\right.$ $=17 \%$; significance $\mathrm{F}=0.3$ ).

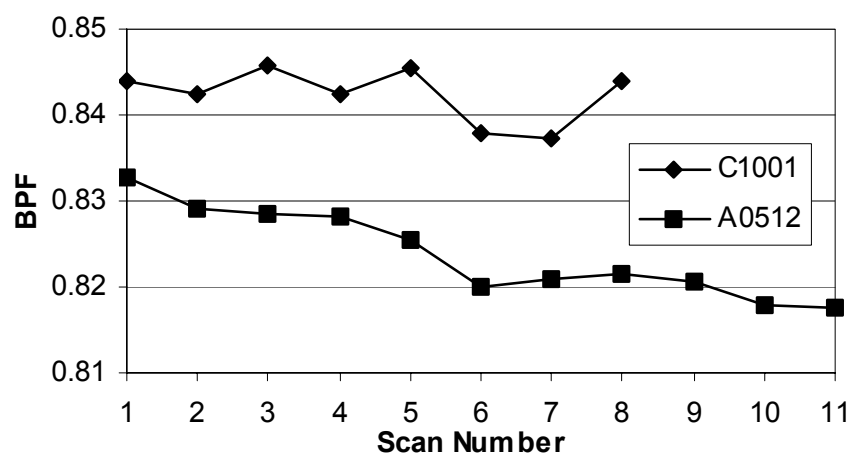

Fig 8. Brain Parenchymal Fraction versus scan number

The BPF results calculated using a $3 \times 3$ dilation kernel over the brain mask for patient $\mathrm{C} 1001$ (mean $\mathrm{BPF}=0.8414$ ) are almost identical to the BPF calculated from the non-dilated mask (mean $\mathrm{BPF}=0.8424$ ). The results for the eroded brain mask show slightly elevated $\mathrm{BPF}$ values (mean $\mathrm{BPF}=0.847$ ) because the CSF was eroded away.

\section{DISCUSSION}

\section{A. Intra-dural volume}

The IDV measurements for the two patients are shown in Fig. 2. We would expect a patient's IDV to be nearly constant. The relatively large variation in brain volume recorded for patient A0512 is due to the fact that the top 3 slices of the brain are not included in these datasets. The data therefore varies according to the position of the patient in the scanner. The patient $\mathrm{C} 1001$ has the complete brain imaged, and shows a much lower coefficient of variation for the IDV $(1.2 \%$ for $\mathrm{A} 0512$, and $0.49 \%$ for $\mathrm{C} 1001$ ).

Most researchers do not report the scan-rescan reproducibility of the intra-dural volume measurement, focusing instead on the fraction of brain in the observed measurements. Chen has reported scan-rescan reproducibility of two brain volume measurements on two volunteers, where the data was acquired with $1.5 \mathrm{~mm}$ thick slices [4]. In one of these cases the difference between two scans was $0.6 \%$. However, segmenting MR head images with $5 \mathrm{~mm}$ thick slices is notoriously subject to partial volume effects, so our results for patient C1001 are entirely reasonable.

\section{B. Sensitivity of volume to CSF ratio image threshold}

The ratio image threshold experiments for patient C1001 shown in Fig. 5 and Fig. 6 show that the CSF volume measurements vary considerably (from $120 \mathrm{~mL}-220 \mathrm{~mL}$ ). From a visual inspection of the CSF masks, it is seen that at a ratio 
threshold of 0.65 the CSF deep in the brain sulci is not segmented, probably due to partial volume effects. The apparent increase in volume of CSF by raising the ratio threshold from 0.65 to 0.75 is mainly due to the inclusion of extra border pixels adjacent to the edges of the CSF pixels. However, above a ratio threshold of 0.75 , other non-CSF tissue is being incorrectly classified as CSF. The sensitivity of the CSF volume to the threshold means that the absolute value of the CSF is likely to be variable, particularly if a manual method for outlining the CSF were used. However, our automatic method is $100 \%$ reproducible, and for a ratio of 0.75 , it appears that all the CSF is correctly segmented.

It can be seen from Fig. 5 that the CSF volume for a given ratio threshold follows a consistent pattern. We have used the ratio threshold of 0.75 successfully in the segmentation of many patient data sets [7], and others also have observed consistent CSF segmentations using T2 and PD data sets [3]. Therefore, for further studies we chose the ratio 0.75 for use in calculating the CSF volumes.

As noted in [2], the BPF is a more stable and repeatable measure than the absolute IDV or CSF volume measures. Rudick et al. speculate that this is due to the fact that errors introduced during repeat acquisitions are present in both the IDV and CSF and therefore counteract each other. The BPF is shown in Fig. 6 for different ratio thresholds. Note that as for the CSF volume, if the same ratio threshold is used for each time-series scan, the BPF follows the same trend.

\section{Sensitivity of VCSF measurement to patient position}

Figure 7 shows that the measured volume of VCSF varies between scans $(\mu=53.96 \mathrm{~mL}, \sigma=2.60 \mathrm{~mL}$, coefficient of variation $=4.8 \%$ ), whereas the coefficient of variation for the total CSF is $2.8 \%$. We hypothesize that the slice thickness of $5 \mathrm{~mm}$ causes incorrect image sampling of the complex 3D ventricle structure (shown in Fig. 1); the variation is not due to the manual segmentation of the VCSF from the CSF. The variation of the observed VCSF volumes suggests that brain atrophy measurements based largely on VCSF volumes derived from thick MR slices must be considered with caution. However, because the VCSF accounts for only about $25 \%$ of the total CSF, the BPF measure based on the total CSF has only a low variation, as discussed below.

\section{Brain Atrophy Measure}

Figure 8 shows the BPF for the two patients over time. For patient $\mathrm{A} 0215$, the $\mathrm{CV}=0.63 \%$. Note that this data set does not include the whole brain; the mis-registration of patient position causes a higher variance than would be observed for a whole brain BPF measurement. Indeed, for patient $\mathrm{C} 1001$, where the whole brain set is available, the $\mathrm{CV}$ is lower, at $0.38 \%$. This is still higher than others have reported for scan-rescan measurements: Fisher reports a mean $\mathrm{CV}=0.19 \%$ for the $\mathrm{BPF}$ from scan-rescan tests with 12 people, using T2-weighted FLAIR scans with $5 \mathrm{~mm}$ thick slices [9] and Collins reports a mean CV of $0.21 \%$ on scan-rescan tests of 4 normal controls, using $3 \mathrm{~mm}$ slices [10]. However, our results are not directly comparable, because we did not acquire scan-rescan images within a short time period. We expect some atrophy in MS patients to occur over time; Rudick reports a drop of $0.7 \%$ per year in placebo patients [2], Jones reports a drop of $0.84 \%$ [3], and Collins reports a drop of $1.8 \%$ for relapsing/remitting MS patients [10]. Our patient A0512 shows a drop of $1.6 \%$ over the whole time series of 11 months, consistent with these findings.

It is encouraging to see that the BPF is almost unchanged when the initial brain mask is slightly dilated, showing that the $\mathrm{BPF}$ is indeed insensitive to an overestimate of the brain contour. If the brain mask is eroded, the erosion of CSF causes a slightly higher effect on the BPF. Therefore it is prudent to err on the side of a too-large brain mask rather than a too-small brain mask.

\section{CONCLUSION}

We found that for purposes of measuring changes of the BPF over time in a particular patient, the BPF obtained automatically by our image processing techniques does indeed appear to be robust and reproducible, and relatively insensitive to errors in measuring the brain outline.

Future work will include many more patient and normal studies to confirm the sensitivity of the method. We then plan to use the method to determine brain atrophy trends in degenerative neural diseases such as MS and Alzheimer's disease.

\section{ACKNOWLEDGMENT}

Many thanks to Dr. David Li for interesting discussions and advice, and to the UBC Multiple Sclerosis Group for providing the data. Funding was provided by the Canada Natural Science and Engineering Research Council.

\section{REFERENCES}

[1] Simon, J.H. et al. "A longitudinal study of brain atrophy in relapsing Multiple Sclerosis", Neurology, 53(8), pp.139-148, July 1999.

[2] Rudick, R.A. et al. "Use of the brain parenchymal fraction to measure whole brain atrophy in relapsing-remitting MS", Neurology, 53(8), pp.16981999, Nov. 1999.

[3] Jones, C. et al., "Atrophy Measurements in Multiple Sclerosis", Proceedings of ISMRM, (9), page 1414, April 2001.

[4] Chen D. et al. "A new method for quantitative analysis of Multiple Sclerosis using MR images", Proceedings of SPIE Medical Imaging, (4319), (in press) Feb. 2001.

[5] Atkins, M.S. and Mackiewich, Blair T., "Fully Automatic Segmentation of the Brain in MRI", IEEE Trans. on Medical Imaging. 17(1), pp. 98-107, Feb. 1998.

[6] Atkins, M.S. and Mackiewich, Blair T., "Fully Automatic Hybrid Segmentation of the Brain", Bankman., ed., Handbook of Medical Imaging, Academic Press: San Diego, California, Chapter 11, pp. 171-183, 2000.

[7] Krishnan, K. and Atkins, M.S., "Segmentation of Multiple Sclerosis Lesions in MRI - An Image Analysis Approach", Proceedings of SPIE Medical Imaging, (3338), pp. 1106-1116, Feb. 1998.

[8] Tory, M.K., Möller, T., and M.S. Atkins, "Visualization of Time-Varying MRI Data for MS Lesion Analysis", Proceedings of SPIE Medical Imaging, (4319), (in press) Feb. 2001.

[9] Fisher, E. et al., "Automated Calculation of Whole Brain Atrophy from Magnetic Resonance Images for Monitoring Multiple Sclerosis", Neurology, (52):A352, 1999.

[10] Collins D.L. et al., "Automated estimation of brain volume in MS with BICCR”, Information Processing in Medical Imaging, to appear, June 2001. 